\title{
A General Family of Multivariable Digital Lattice Filters
}

\author{
P. P. VAIDYANATHAN, MEMBER, IEEE, AND SANJIT K. MITRA, FELLOW, IEEE
}

\begin{abstract}
Lattice structures are developed for the realization of $m$-input $p$-output discrete-time all-pass transfer matrices $\boldsymbol{H}(z)$, given in the form of a right matrix-fraction description (MFD): $H(z)=$ $N(z) D^{-1}(z)$. The procedure is based on the generation of a sequence of all pass matrices of successively decreasing order, by matrix LBR two-pair extraction. Two cases are distinguished: the first case is when none of the intermediate allpass matrices is degenerate. For this case, the resulting structures are in the form of a cascade of matrix two-pairs separated by vector delays, with each two-pair being a multi-input multi-output digital filter structure characterized by an orthogonal transfer matrix of dimension $(m+p) \times(m+p)$. The structures are in general either completely controllable or completely observable, depending upon the location of the delay elements. The synthesis technique also leads to a procedure for obtaining the greatest common right divisor between the polynomial matrices involved in the MFD. The results are extended to the cascaded-lattice synthesis of arbitrary stable transfer matrices by an embedding process. The developments of this paper automatically place in evidence a procedure for testing the stability of a transfer matrix. A special case of the resulting structures when $p=m-1$ gives rise to the well-known Gray-Markel digital lattice structures, whereas another special case with $p=2$ and $m=1$ leads to certain recently reported orthogonal digital fitlers. The second case, where some of the intermediate allpass matrices are degenerate, is handled separately, leading to a modified form of cascaded-multivariable lattice structures.
\end{abstract}

\section{INTRODUCTION}

$\mathrm{T}$ HE generation of digital lattice filters for scalar all-pass transfer functions of the form

$$
H(z)=z^{-N} B\left(z^{-1}\right) / B(z)
$$

can be interpreted from a number of different, but related, viewpoints. For example, the synthesis can be related to the theory of orthogonal polynomials [1], [2]. The synthesis is also inherently related to the well-known Levinson's recursion [4] and can be viewed as an algorithm for the inversion of a Toeplitz matrix, which also arises in the theory of linear prediction. The extension of the linear-prediction concepts and the Levinson's recursion scheme to vector processes is well known [5], [6]. The mathematical structure offered by such extensions has already been used by some authors for the generation of digital lattice filters with multiple inputs and outputs [7], [8]. For example, Henrot and Mullis [8] have judiciously adapted the

Manuscript received November 22, 1984; revised July 17, 1985. This work was supported in part by the National Science Foundation under Grant ECS 82-18310, in part by CALTECH's Programs in Advanced Technology Grant, and in part by the National Science Foundation under Grant ECS 84-04245.

P. P. Vaidyanathan is with the Department of Electrical Engineering, California Institute of Technology, Pasadena, CA 91125.

S. K. Mitra is with the Department of Electrical and Computer Engineering, University of California, Santa Barbara, CA 93106.
Levinson algorithm for the synthesis of "orthogonal digital filters" with one input and two outputs, with the ultimate purpose of realizing an arbitrary scalar transfer function, in the form of a cascade of a generalization of the wellknown lattice structures. Some of these lattice structures are related, in turn, to the concept of losslessness, and the relation between Levinson's filters and lossless functions itself is known [9], [23], [24]. Finally, the relation between cascade synthesis of lossless functions and the Nevanlinna-Pick interpolation problem has also been studied [22] by Delsarte et al.

The purpose of this paper is to develop a synthesis procedure for a $m$-input $p$-output Lossless Bounded Real (LBR) transfer matrix, given in the form of a right Matrix Fraction Description (MFD) [10], [17]:

$$
\boldsymbol{H}(z)=\boldsymbol{N}(z) D^{-1}(z)
$$

where $\boldsymbol{N}(z)$ and $\boldsymbol{D}(z)$ are $p \times m$ and $m \times m$ polynomial matrices, respectively. The synthesis procedure leads to a cascaded vector lattice structure, with adjacent lattice building blocks being separated by vector delays as shown in Fig. 1. Each lattice building block has an orthogonal transfer matrix, and is an extension of the $2 \times 2$ lattice transfer matrix [1]. The synthesis procedure is based entirely on the LBR-extraction approach [11], and is developed in a self-contained manner. Basically, given an LBR transfer matrix $\boldsymbol{G}_{N}(z)$, we generate a sequence of LBR transfer matrices $\boldsymbol{G}_{N-1}(z), \boldsymbol{G}_{N-2}(z), \ldots$ by successive LBR two-pair extraction, such that $\boldsymbol{G}_{m-1}(z)$ has lower order than $\boldsymbol{G}_{m}(z)$. We distinguish two main cases. The first one is where all $\boldsymbol{G}_{m}(z)$ are "nondegenerate" (to be defined) allpass matrices. The second case is where some of the intermediate allpass functions $\boldsymbol{G}_{m}(z)$ are degenerate in a certain sense. The second case mentioned above is handled separately in the Appendix, so that the main text has a smooth flow. Unless mentioned otherwise, comments in the main text pertain to the first case. Even though the synthesis procedure can possibly be related to a suitably modified Levinson's recursion and matrix orthogonal polynomial theory [12], no attempt has been made here to explicitly analyze such relationships. One motivation for an independent derivation of the lattice structures, among others, is that the existence (and derivation) does not seem to follow in an obvious manner from known thcory, for arbitrary $p$ and $m(p \neq m$ in general $)$. The structures developed in this paper are such that the well-known Gray-Markel normalized-lattice structures are obtained 


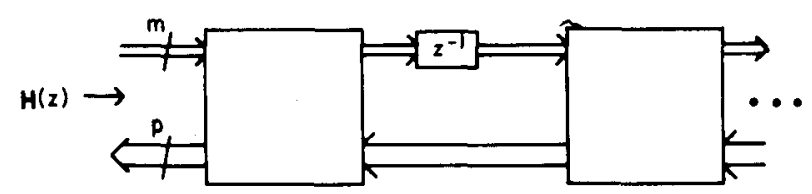

Fig. 1. Cascaded lattice for $p \times m \boldsymbol{H}(z)$.

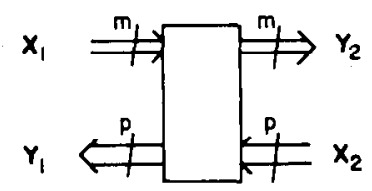

Fig. 2. The matrix two-pair. with $p=m=1$. Similarly, structures developed in [8] and [13] result with $p=2$ and $m=1$.

In Section II we review some basic concepts and notations. Section III deals with the "order-reduction problem," based on the LBR matrix two-pair extraction approach. This is the key for the entire synthesis procedure. A numerical example is included here to illustrate the synthesis. In Section IV several properties of the structures are developed, including inherent stability, controllability, observability, and possible minimality. In Section $V$ it is shown that, with LBR transfer matrices given in the form of nonminimal MFD's, the synthesis procedure directly places in evidence a greatest common right divisor. (In fact, the synthesis terminates in a "prematured manner," and the "controllable form" automatically becomes a minimal realization, even though the given MFD is nonminimal.) Finally, Section VI outlines the synthesis of arbitrary stable transfer matrices in the form of a cascaded lattice.

\section{Preliminaries}

\section{Notations}

In this paper, superscript $t$ stands for matrix transposition, whereas superscript dagger $(\dagger)$ stands for transposition followed by complex conjugation. Boldface letters denote matrices and vectors. The tilde accent stands for transposition followed by reciprocation of functional arguinent; for example, $\tilde{\boldsymbol{H}}(z)=\boldsymbol{H}^{t}\left(z^{-1}\right)$. The notation $\boldsymbol{A} \leqslant \boldsymbol{B}$ (where $\boldsymbol{A}$ and $\boldsymbol{B}$ are square matrices of equal dimensions) is abbreviation for " $\boldsymbol{B}-\boldsymbol{A}$ is positive semidefinite." Similarly, $\boldsymbol{A}<\boldsymbol{B}$ means " $\boldsymbol{B}-\boldsymbol{A}$ is positive definite." $\boldsymbol{I}_{m}$ denotes the identity matrix of dimension $m \times m$. For a (real symmetric) positive definite matrix $\boldsymbol{P}$, we define its square root $\boldsymbol{P}^{1 / 2}$ according to the factorization $\boldsymbol{P}=\boldsymbol{P}^{1 / 2} \boldsymbol{P}^{t / 2}$ where $\boldsymbol{P}^{t / 2}$ is the transpose of $\boldsymbol{P}^{1 / 2}$.

An $m$-input $p$-output lincar-shift invariant discrete-time system can be described by a $p \times m$ transfer matrix $\boldsymbol{H}(z)$. In this paper, we deal exclusively with right matrix fraction descriptions (MFD) [10], [17] of $\boldsymbol{H}(z)$, as in (2). The MFD is said to be "irreducible" if $N(z)$ and $D(z)$ are rightcoprime, i.e., any greatest common right divisor is an $m \times m$ unimodular matrix-polynomial. ${ }^{1} \mathrm{~A}$ matrix transfer function $\boldsymbol{H}(z)$ with rational entries is said to be LBR (lossless bounded real [14]) if (a) $\boldsymbol{H}(z)$ is a real matrix for real $z$, (b) $\boldsymbol{H}(z)$ is "stable," i.e., all zeros of $\operatorname{det} \boldsymbol{D}(z)$ in an irreducible MFD are strictly within the unit circle, and (c) $\boldsymbol{H}^{\dagger}\left(e^{j \omega}\right) \boldsymbol{H}\left(e^{j \omega}\right)$ is the $m \times m$ identity matrix $\boldsymbol{I}_{m}$. It can be shown that a $p \times m$ transfer matrix $\boldsymbol{H}(z)$ cannot be LBR if $p<m$ (see Appendix A1). We, therefore, assume $p \geqslant m$.

${ }^{1} \mathrm{~A}$ unimodular matrix polynomial $W(z)$ is a square matrix, with $\operatorname{det} \boldsymbol{W}(z)=$ constant $\neq 0$.
Note that "scattering matrices" of continuous-time lossless multiports satisfy the LBR property, but are always square matrices (i.c., $p=m$ ). The synthesis methods we develop in this paper, however, hold for arbitrary $p \geqslant m$.

An important property of an LBR transfer matrix is the following equality:

$$
\boldsymbol{H}^{t}\left(z^{-1}\right) \boldsymbol{H}(z)=\boldsymbol{I}_{m}, \quad \text { for all } z .
$$

Another extremely important inequality, satisfied by a $p \times m$ LBR transfer matrix $\boldsymbol{H}(z)$, is the following:

$$
\boldsymbol{H}^{\dagger}\left(z_{0}\right) \boldsymbol{H}\left(z_{0}\right) \leqslant \boldsymbol{I}_{m}, \quad \text { for }\left|z_{0}\right|>1 .
$$

A proof, based only on linear-system theory arguments, is included in Appendix A2. (We feel that the inequality is not quite obvious because, a $p \times m$ LBR matrix cannot be obtained by straightforward transformations of impedance matrices, which are square, into the scattering domain. See also [20], [21].) As we shall see, the above inequality plays a crucial role in our lattice-generation procedure. It can be shown that, if the inequality of (3b) is not strict for some $z_{0}$ in $\left|z_{0}\right|>1$, i.e, if there exists a vector $\boldsymbol{V} \neq \mathbf{0}$ such that

$$
\boldsymbol{V}^{\dagger} \boldsymbol{H}^{\dagger}\left(z_{0}\right) \boldsymbol{H}\left(z_{0}\right) \boldsymbol{V}=\boldsymbol{V}^{\dagger} \boldsymbol{V}, \quad\left|z_{0}\right|>1
$$

then $\boldsymbol{H}(z) \boldsymbol{V}$ is constant for all $z$. In other words, $\boldsymbol{H}(z)$ is memoryless ${ }^{2}$ in the direction $\boldsymbol{V}$. Elaborations of these details are included in Appendix A2. In this paper, we have frequent occasion to use the parameter

$$
\mathscr{K}=\boldsymbol{H}(\infty)
$$

where $\boldsymbol{H}(z)$ is a $p \times m$ BR matrix. Many of the steps involved in our synthesis procedures are valid only if the matrix $\left(I_{m}-\mathscr{K}^{\imath} \mathscr{K}\right)$ is nonsingular. This in turn is true if and only if the BR function $H(z)$ is nondegenerate, as shown in Appendix A2. The situations where $\left(I_{m}-\mathscr{K}^{t} \mathscr{K}\right)$ has a rank-defect (i.e., is singular) is handled separately in Appendix A4, so as to maximize the clarity of the main text.

A matrix digital two-pair is a $(m+p)$-input $(m+p)$ output system, as shown in Fig. 2, with a transfer matrix description:

$$
\begin{aligned}
& \boldsymbol{Y}(z)=\left[\begin{array}{l}
\boldsymbol{Y}_{1}(z) \\
\boldsymbol{Y}_{2}(z)
\end{array}\right]=\left[\begin{array}{ll}
\boldsymbol{T}_{11}(z) & \boldsymbol{T}_{12}(z) \\
\boldsymbol{T}_{21}(z) & T_{22}(z)
\end{array}\right]\left[\begin{array}{l}
X_{1}(z) \\
X_{2}(z)
\end{array}\right] \\
& =\mathrm{T}(z) \boldsymbol{X}(z) \text {. }
\end{aligned}
$$

Here $\boldsymbol{X}_{1}(z)$ and $\boldsymbol{X}_{2}(z)$ are vectors of dimensions $m$ and $p$, respectively. Likewise, $Y_{1}(z)$ and $Y_{2}(z)$ are vectors of dimensions $p$ and $m$, respectively.

\footnotetext{
${ }^{2}$ In this paper, a transfer matrix that is not memoryless in any direction is termed nondegenerate. If it is memoryless in some direction, we call it degenerate.
} 
The two-pair can also be described by the chain parameters:

$$
\left[\begin{array}{c}
X_{1}(z) \\
Y_{1}(z)
\end{array}\right]=\left[\begin{array}{ll}
A(z) & B(z) \\
C(z) & D(z)
\end{array}\right]\left[\begin{array}{c}
Y_{2}(z) \\
X_{2}(z)
\end{array}\right] .
$$

The two descriptions are related through:

$$
\begin{array}{lll}
\boldsymbol{A}=\boldsymbol{T}_{21}^{-1}, & \boldsymbol{B}=-\boldsymbol{T}_{21}^{-1} \boldsymbol{T}_{22} \\
\boldsymbol{C}=\boldsymbol{T}_{11} \boldsymbol{T}_{21}^{-1}, & \boldsymbol{D}=\boldsymbol{T}_{12}-\boldsymbol{T}_{11} \boldsymbol{T}_{21}^{-1} \boldsymbol{T}_{22}
\end{array}
$$

and

$$
\begin{array}{ll}
\boldsymbol{T}_{11}=\boldsymbol{C A}^{-1}, & \boldsymbol{T}_{12}=\boldsymbol{D}-\boldsymbol{C A}^{-1} \boldsymbol{B} \\
\boldsymbol{T}_{21}=\boldsymbol{A}^{-1}, & \boldsymbol{T}_{22}=-\boldsymbol{A}^{-1} \boldsymbol{B} .
\end{array}
$$

Note that $\boldsymbol{A}$ and $\boldsymbol{T}_{21}$ are square matrices. (The inverses of $\boldsymbol{A}$ and $\boldsymbol{T}_{21}$ are assumed to exist, in (6), (7). In Section III we indicate situations where these inverses do not exist, and also indicate how these can be handled.) Thus a matrix digital two-pair is essentially a digital two-pair [18] such that some or all of the chain parameters are matrices or vectors. Given a $p \times m$ transfer matrix $\boldsymbol{G}_{k}(z)$, the "extraction" of a matrix digital two-pair, leading to a remainder $\boldsymbol{G}_{k-1}(z)$ is sketched in Fig. 3. The matrices $\boldsymbol{G}_{k}(z)$ and $\boldsymbol{G}_{k-1}(z)$ are related by

$$
\begin{aligned}
\boldsymbol{G}_{k-1}(z) & =\left(\boldsymbol{D}-\boldsymbol{G}_{\boldsymbol{k}} \boldsymbol{B}\right)^{-1}\left(\boldsymbol{G}_{\boldsymbol{k}} \boldsymbol{A}-\boldsymbol{C}\right) \\
\boldsymbol{G}_{k}(z) & =\left(\boldsymbol{C}+\boldsymbol{D} \boldsymbol{G}_{k-1}\right)\left(\boldsymbol{A}+\boldsymbol{B} \boldsymbol{G}_{k-1}\right)^{-1}
\end{aligned}
$$

where $A, B, C, D$ are the chain parameters of the extracted two-pair. Note that (8) and (9) are equivalent. Equation (8) is suitable for left-MFD descriptions, whereas (9) is suitable for right-MFD descriptions.

A matrix two-pair is said to be LBR if its transfer matrix $\mathrm{T}(z)$ is LBR. In terms of the chain parameters, the LBR property of a stable two-pair (with $\mathbf{T}(z)$ real for real $z$ ) is equivalent to the following set of conditions:

$$
\begin{aligned}
\tilde{\boldsymbol{A}}(z) \boldsymbol{A}(z)-\tilde{\boldsymbol{C}}(z) \boldsymbol{C}(z) & =\boldsymbol{I}_{m} \\
\tilde{\boldsymbol{D}}(z) \boldsymbol{D}(z)-\tilde{\boldsymbol{B}}(z) \boldsymbol{B}(z) & =\boldsymbol{I}_{p} \\
\tilde{\boldsymbol{A}}(z) \boldsymbol{B}(z) & =\tilde{\boldsymbol{C}}(z) \boldsymbol{D}(z) .
\end{aligned}
$$

This can be compactly expressed [19] in the form

$$
\tilde{\Theta}(z) J \Theta(z)=J
$$

where $\Theta(z)$ is the chain matrix

$$
\Theta(z)=\left[\begin{array}{ll}
A(z) & B(z) \\
C(z) & D(z)
\end{array}\right]
$$

and

$$
\boldsymbol{J}=\left[\begin{array}{cc}
\boldsymbol{I}_{m} & \boldsymbol{O} \\
\boldsymbol{O} & -\boldsymbol{I}_{p}
\end{array}\right]
$$

Moreover, the inequality of (3b) which holds for an LBR two-pair transfer matrix can be re-expressed in terms of the chain matrix $\Theta(z)$ as

$$
\Theta^{\dagger}(z) J \Theta(z) \geqslant J, \quad|z| \geqslant 1 \text {. }
$$

Note that a constant matrix two-pair is LBR if and only

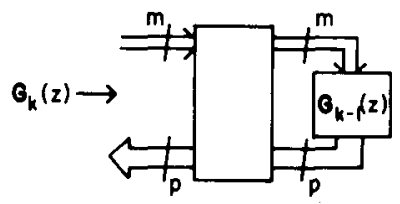

Fig. 3. Matrix two-pair extraction.

if the (constant) transfer matrix $\mathbf{T}$ is orthogonal i.e., $\mathbf{T}^{\prime} \mathbf{T}=\boldsymbol{I}_{m+p}$.

\section{The LBR Extraction SteP}

In this section, we solve the central problem that is basic to our lattice synthesis. Given a $p \times m$ LBR transfer matrix $\boldsymbol{G}_{k}(z)$ in right-MFD form

$$
\boldsymbol{G}_{k}(z)=\boldsymbol{N}_{k}(z) \boldsymbol{D}_{k}^{-1}(z) \text {. }
$$

We wish to "extract" a constant matrix LBR two-pair with chain parameters $\boldsymbol{A}_{k}, \boldsymbol{B}_{k}, \boldsymbol{C}_{k}, \boldsymbol{D}_{k}$ such that the remainder is a $p \times m$ LBR matrix of the form

$$
z^{-1} \boldsymbol{G}_{k-1}(z)=z^{-1} \boldsymbol{N}_{k-1}(z) \boldsymbol{D}_{k-1}^{-1}(z)
$$

where $\boldsymbol{N}_{k}(z), \boldsymbol{D}_{k}(z), \boldsymbol{N}_{k-1}(z), \boldsymbol{D}_{k-1}(z)$ are polynomial matrices given by

$$
\begin{aligned}
\boldsymbol{N}_{k}(z) & =\sum_{i=0}^{k} \boldsymbol{A}_{k i} z^{-i} \\
\boldsymbol{D}_{k}(z) & =\sum_{i=0}^{k} \boldsymbol{B}_{k i} z^{-i} \\
z^{-1} \boldsymbol{N}_{k-1}(z) & =z^{-1} \sum_{i=0}^{k-1} \boldsymbol{A}_{k-1, i} z^{-i} \\
\boldsymbol{D}_{k-1}(z) & =\sum_{i=0}^{k-1} \boldsymbol{B}_{k-1, i} z^{-i} .
\end{aligned}
$$

Here $\boldsymbol{A}_{j i}$ are $p \times m$ real matrices, whereas $\boldsymbol{B}_{j i}$ are $m \times m$ real matrices. (According to linear-systems terminology [10], the remainder $z^{-1} \boldsymbol{G}_{k-1}(z)$ is "strictly" proper, i.e., it tends to 0 as $z \rightarrow \infty$.) Thus $\boldsymbol{G}_{k}(z)$ is an MFD of degree $k$, (i.e., the polynomial matrices involved have degree $k$ ) whereas $\boldsymbol{G}_{k-1}(z)$ has degree $k-1$. Given a $p \times m$ LBR matrix $G_{N}(z)$ :

$$
G_{N}(z)=N_{N}(z) D_{N}^{-1}(z)
$$

if the above extraction process is performed $N$ times, then $\boldsymbol{G}_{N}(z)$ can be realized as a cascade of matrix-LBR twopairs, with successive two-pairs separated by vector delays (see Fig. 4). We assume that $N_{N}(z)$ and $D_{N}(z)$ have the form

$$
N_{N}(z)=\sum_{i=0}^{N} A_{N i} z^{-i}, \quad D_{N}(z)=\sum_{i=0}^{N} B_{N i} z^{-i}
$$

with $\boldsymbol{B}_{N 0}=\boldsymbol{D}_{N}(\infty)$ being nonsingular. (In other words, $\boldsymbol{D}_{N}(z)$ is a regular polynomial matrix [15].) Now with $\boldsymbol{B}_{N 0}$ assumed to be nonsingular, we might as well take it to be $\boldsymbol{I}_{\boldsymbol{m}}$. As we shall see, if we assume $\boldsymbol{B}_{k 0}=\boldsymbol{I}_{m}$, the remainder $\boldsymbol{G}_{k-1}(z)$ has the property $\boldsymbol{B}_{k-1,0}=\boldsymbol{I}_{m}$. 


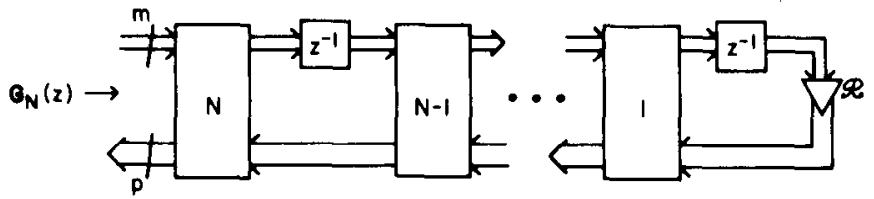

Fig. 4. Cascaded lattice realization of $G_{N}(z)$.

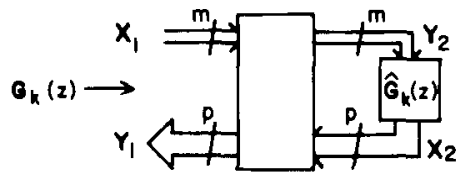

Fig. 5. The order reduction problem.

\section{The Order-Reduction Problem}

Given an LBR MFD $\boldsymbol{G}_{k}(z)$ as described in (11) and (13) with $D_{k}(\infty)=I_{m}$, we first examine the following problem: extract a two-pair with constant chain parameters $\{\boldsymbol{A}, \boldsymbol{B}, \boldsymbol{C}, \boldsymbol{D}\}$ such that the remainder $\hat{\boldsymbol{G}}_{k}(z)$ (Fig. 5) is of the form

$$
\begin{aligned}
& \hat{\boldsymbol{G}}_{k}(z)=\hat{\boldsymbol{N}}_{k}(z) \hat{\boldsymbol{D}}_{k}^{-1}(z) \\
& \hat{\boldsymbol{N}}_{k}(z)=z^{-1} \sum_{i=0}^{k-1} \hat{\boldsymbol{A}}_{k, i} z^{-i}, \quad \hat{\boldsymbol{D}}_{k}(z)=\sum_{i=0}^{k-1} \hat{\boldsymbol{B}}_{k, i} z^{-i} .
\end{aligned}
$$

In other words, let us temporarily relax the restriction that the two-pair and the remainder should be LBR, and concentrate only on order reduction. We assume $\boldsymbol{D}=\boldsymbol{I}_{p}$, and show that $\boldsymbol{A}, \boldsymbol{B}$, and $\boldsymbol{C}$ can be determined such that the order-reduction is accomplished. It is shown in Appendix A3 that $\boldsymbol{G}_{k}$ and $\hat{\boldsymbol{G}}_{k}$ are related as

$$
\begin{aligned}
& \boldsymbol{D}_{k}=\boldsymbol{A} \hat{\boldsymbol{D}}_{k}+\boldsymbol{B} \hat{\boldsymbol{N}}_{k} \\
& \boldsymbol{N}_{k}=\boldsymbol{C} \hat{\boldsymbol{D}}_{k}+\boldsymbol{D} \hat{\boldsymbol{N}}_{k} .
\end{aligned}
$$

Equations (19) and (20) are true within an arbitrary common right-divisor, which can be cancelled in the right MFD of (11).

Now, we are trying to find the chain parameters $A, B, C, D$ such that $\hat{N}_{k}(z)$ is forced to have the form shown in (18). This can be accomplished simply by picking $C$ so that it satisfies

$$
C \hat{D}_{k}(\infty)=N_{k}(\infty)
$$

From (19) and (20) we also get

$$
\hat{D}_{k}(z)=(A-B C)^{-1}\left[D_{k}(z)-B N_{k}(z)\right] \text {. }
$$

In writing (22), it is implicitly assumed that the matrix $(\boldsymbol{A}-\boldsymbol{B C})$ is nonsingular. It can be shown (see comments following 32) that $(\boldsymbol{A}-\boldsymbol{B C})$ is nonsingular if and only if $\boldsymbol{G}_{k}(z)$ is nondegenerate.

In order to reduce the degree of $\hat{D}_{k}(z)$ to $k-1$, it is sufficient to choose $B$ so that

$$
\left[z^{k} \boldsymbol{D}_{k}(z)\right]_{z=0}=\boldsymbol{B}\left[z^{k} \boldsymbol{N}_{k}(z)\right]_{z=0} \text {. }
$$

Moreover, if $\boldsymbol{G}_{k}(z)$ is LBR, then an equality analogous to (3a) holds, hence

$$
D_{k}^{t}(\infty)\left[z^{k} D_{k}(z)\right]_{z=0}=\boldsymbol{N}_{k}^{t}(\infty)\left[z^{k} \boldsymbol{N}_{k}(z)\right]_{z=0} \text {. }
$$

Recall that we have assumed $D_{k}(\infty)=I_{m}$. As a result, the choice of

$$
\boldsymbol{B}=\boldsymbol{N}_{k}^{t}(\infty)
$$

ensures the order reduction. The choice of $\boldsymbol{A}$ is flexible, and by choosing $\boldsymbol{A}=\boldsymbol{I}_{m}$, we arrive at a remainder for which $\hat{D}_{k}(\infty)=\hat{B}_{k, 0}=\boldsymbol{I}_{m}$. The choice of $\boldsymbol{C}$ is governed by (21). In summary, the following set of chain parameters:

$$
\boldsymbol{A}=\boldsymbol{I}_{m}, \quad \boldsymbol{B}=\mathscr{K}^{\prime}, \quad C=\mathscr{X}, \quad D=I_{p}
$$

with the $p \times m$ matrix $\mathscr{K}$ given by

$$
\mathscr{K}=G_{k}(\infty)=N_{k}(\infty)
$$

leads to the desired order reduction. However, the two-pair described by the above set of chain parameters is not LBR because (10a) and (10b) are not satisfied. In fact, instead of (10a)-(10c) we actually have

$$
\begin{aligned}
\tilde{A A}-\tilde{C} C & =I_{m}-\mathscr{K}^{t} \mathscr{K} \\
\tilde{D} D-\tilde{B} B & =I_{p}-\mathscr{K} \mathscr{K}^{t} \\
\tilde{A} B & =\mathscr{K}^{t}=\tilde{C} D .
\end{aligned}
$$

Accordingly, we should "scale" the chain parameters so that the extracted two-pair becomes LBR; this is done next. Now $\boldsymbol{G}_{k}(z)$ being LBR, the $p \times m$ matrix $\mathscr{K}$ defined by (27) satisfies

$$
\mathscr{K}^{t} \mathscr{K} \leqslant \boldsymbol{I}_{m}
$$

by (3b). The following inequality, therefore, follows

$$
\mathscr{K} \mathscr{K}^{t} \leqslant \boldsymbol{I}_{p}
$$

because the nonzero eigenvalues of $\mathscr{K}^{t} \mathscr{K}$ and $\mathscr{K} \mathscr{K}^{t}$ are the same. Consequently, the matrices on the right side of (28a) and (28b) are positive semidefinite and can be factorized as

$$
\begin{aligned}
\boldsymbol{I}_{m}-\mathscr{K}^{t} \mathscr{K} & =\left[\boldsymbol{I}_{m}-\mathscr{K}^{t} \mathscr{K}\right]^{1 / 2}\left[\boldsymbol{I}_{m}-\mathscr{K}^{t} \mathscr{K}^{t / 2}\right. \\
\boldsymbol{I}_{p}-\mathscr{K} \mathscr{K}^{t} & =\left[\boldsymbol{I}_{p}-\mathscr{K} \mathscr{K}^{t}\right]^{1 / 2}\left[\boldsymbol{I}_{p}-\mathscr{K} \mathscr{K}^{t}\right]^{t / 2} .
\end{aligned}
$$

Recall that, if $\boldsymbol{G}_{k}(z)$ is nondegenerate (Appendix A2), then (29) and (30) are strict inequalities, and hence $\left(I_{m}-\mathscr{K}^{t} \mathscr{K}\right)$ and $\left(I_{p}-\mathscr{K} \mathscr{K}^{t}\right)$ are strictly positive definite. The inverses of the matrices in (31), (32) then exist. Thus $A-B C$ is nonsingular, and (22) is valid. Thus if the chain parameters are redefined as

$$
\begin{aligned}
\boldsymbol{A} & =\left[\boldsymbol{I}_{m}-\mathscr{K}^{t} \mathscr{K}^{t / 2},\right. & \boldsymbol{B} & =\mathscr{K}^{t}\left[\boldsymbol{I}_{p}-\mathscr{K} \mathscr{K}^{t}\right]^{-t / 2} \\
\boldsymbol{C} & =\mathscr{K}\left[\boldsymbol{I}_{\boldsymbol{m}}-\mathscr{K}^{t} \mathscr{K}\right]^{-t / 2}, & \boldsymbol{D} & =\left[\boldsymbol{I}_{p}-\mathscr{K} \mathscr{K}^{t}\right]^{-t / 2}
\end{aligned}
$$

the extracted two-pair is indeed LBR, and the new remainder is

$$
z^{-1} G_{k-1}(z)=z^{-1} N_{k-1}(z) D_{k-1}^{-1}(z)
$$




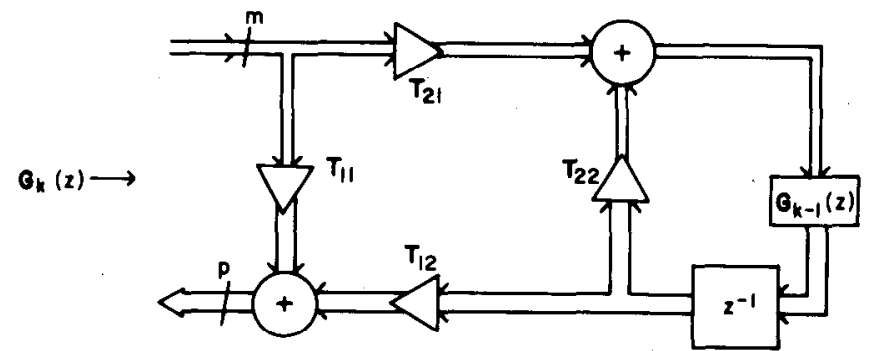

Fig. 6. The LBR matrix two-pair lattice

where

$$
z^{-1} \boldsymbol{N}_{k-1}(z)=D^{-1} \hat{\boldsymbol{N}}_{k}(z) ; \quad \boldsymbol{D}_{k-1}(z)=\boldsymbol{A}^{-1} \hat{\boldsymbol{D}}_{k}(z)
$$

with $\boldsymbol{A}$ and $\boldsymbol{D}$ being as defined in (33). Note that the LBR two-pair described by (33) has transfer matrix:

$$
\mathrm{T}_{k}=\left[\begin{array}{cc}
\mathscr{K} & \left(I_{p}-\mathscr{K} \mathscr{K}^{t}\right)^{1 / 2} \\
\left(\boldsymbol{I}_{m}-\mathscr{K}^{t} \mathscr{K}\right)^{t / 2} & -\left(\boldsymbol{I}_{m}-\mathscr{K}^{t} \mathscr{K}\right)^{t / 2} \mathscr{K}^{t}\left(I_{p}-\mathscr{K} \mathscr{K}^{t}\right)^{-t / 2}
\end{array}\right]
$$

which can be verified to be orthogonal, as expected. The overall situation is shown in Fig. 6. Notice the resemblance to the normalized Gray-Markel scalar lattice [1]. It is easily shown that $N_{k-1}(z)$ and $D_{k-1}(z)$ have the following explicit expressions:

$$
\begin{aligned}
z^{-1} \boldsymbol{N}_{k-1}(z) & =\left(\boldsymbol{I}_{p}-\mathscr{K} \mathscr{K}^{t}\right)^{-1 / 2}\left[\boldsymbol{N}_{k}(z)-\mathscr{K} \boldsymbol{D}_{k}(z)\right] \\
\boldsymbol{D}_{k-1}(z) & =\left(\boldsymbol{I}_{\boldsymbol{m}}-\mathscr{K}^{t} \mathscr{K}^{-1 / 2}\left[\boldsymbol{D}_{k}(z)-\mathscr{K}^{t} \boldsymbol{N}_{k}(z)\right]\right.
\end{aligned}
$$

where $\mathscr{K}$ is given by (27) and satisfies (29) and (30). This then gives us the formula for computing the remainder function $\boldsymbol{G}_{k-1}(z)$. We emphasize here that, if the LBR matrix $\boldsymbol{G}_{k}(z)$ is degenerate, then $\boldsymbol{I}_{m}-\mathscr{K}^{t} \mathscr{K}$ is singular, and (33) and (36) do not hold. Appendix A4 handles this situation.

Now, from (37b) we have

$$
\boldsymbol{D}_{k-1}(\infty)=\left(\boldsymbol{I}_{m}-\mathscr{K}^{t} \mathscr{K}\right)^{-1 / 2}\left(\boldsymbol{I}_{\boldsymbol{m}}-\mathscr{K}^{\boldsymbol{t}} \mathscr{K}^{\prime}\right) \boldsymbol{D}_{k}(\infty)
$$

Recall that $D_{k}(\infty)=I_{m}$, hence,

$$
D_{k-1}(\infty)=\left(I_{m}-\mathscr{K}^{t} \mathscr{K}\right)^{t / 2}
$$

As long as $\boldsymbol{G}_{k}(z)$ is nondegenerate, $\boldsymbol{D}_{k-1}(\infty)$ is, therefore, nonsingular, so that the earlier recursion step can be repeated.

\section{The Remainder After Extraction}

We wish to repeat the above LBR extraction process by working on $G_{k-1}(z)$ so that, after a finite number of such steps, a complete cascaded lattice is obtained. In order to do this, we need to prove that $G_{k-1}(z)$ is LBR. To this end we observe that

(a) $G_{k-1}(z)$ is real for real $z$.

(b) From Eqn. (8) it can be verified that $\boldsymbol{G}_{k-1}^{\dagger}\left(e^{j \omega}\right) \boldsymbol{G}_{k-1}\left(e^{j \omega}\right)=I_{m}$. This is because the twopair is LBR, and $G_{k}(z)$ itself is LBR.

It, therefore, only remains to show that $\boldsymbol{G}_{k-1}(z)$ is stable, in order to establish that $G_{k-1}(z)$ is LBR.

Recall that $\boldsymbol{G}_{k}(z)=\boldsymbol{N}_{k}(z) \boldsymbol{D}_{k}^{-1}(z)$ is stable; this does not, however, imply that all determinantal zeros of $\boldsymbol{D}_{k}(z)$ are in $|z|<1$. This is because $\left\{N_{k}(z), D_{k}(z)\right\}$ may not be right-coprime, i.e., the MFD may not be irreducible. Thus let

$$
\begin{aligned}
& N_{k}(z)=\bar{N}_{k}(z) \boldsymbol{R}(z) \\
& D_{k}(z)=\bar{D}_{k}(z) \boldsymbol{R}(z)
\end{aligned}
$$

where $\boldsymbol{R}(z)$ is a greatest common right divisor (GCRD) of $\boldsymbol{N}_{k}(z)$ and $\boldsymbol{D}_{k}(z)$, so that $\left\{\overline{\boldsymbol{N}}_{k}(z), \bar{D}_{k}(z)\right\}$ are right coprime. When we say $\boldsymbol{G}_{k}(z)$ is stable, we mean:

$$
\operatorname{det} \bar{D}_{k}\left(z_{0}\right)=0 \Rightarrow\left|z_{0}\right|<1 \text {. }
$$

Now, from (37a) and (37b):

$$
\begin{aligned}
z^{-1} \boldsymbol{N}_{k-1}(z)= & \left(I_{p}-\mathscr{K} \mathscr{K}^{t}\right)^{-1 / 2}\left[\bar{N}_{k}(z)-\mathscr{K} \overline{\boldsymbol{D}}_{k}(z)\right] \boldsymbol{R}(z) \\
\boldsymbol{D}_{k-1}(z)= & \left(\boldsymbol{I}_{m}-\mathscr{K}^{t} \mathscr{K}^{-1 / 2}\right. \\
& \cdot\left[\overline{\boldsymbol{D}}_{k}(z)-\mathscr{K}^{t} \overline{\boldsymbol{N}}_{k}(z)\right] \boldsymbol{R}(z)
\end{aligned}
$$

Thus $\boldsymbol{G}_{k-1}(z)$ is stable provided:

$$
\operatorname{det}\left[\overline{\boldsymbol{D}}_{k}\left(z_{0}\right)-\mathscr{K}^{t} \overline{\boldsymbol{N}}_{k}\left(z_{0}\right)\right]=0 \Rightarrow\left|z_{0}\right|<1 .
$$

Let us assume the contrary, i.e., let

$$
\operatorname{det}\left[\overline{\boldsymbol{D}}_{k}\left(z_{0}\right)-\mathscr{K}^{t} \overline{\boldsymbol{N}}_{k}\left(z_{0}\right)\right]=0, \quad \text { for some }\left|z_{0}\right| \geqslant 1 .
$$

By stability of $\boldsymbol{G}_{k}(z), \overline{\boldsymbol{D}}_{k}\left(z_{0}\right)$ is nonsingular, hence (45) is equivalent to

$$
\operatorname{det}\left[\boldsymbol{I}_{\boldsymbol{m}}-\mathscr{K}^{t} \boldsymbol{G}_{k}\left(z_{0}\right)\right]=0, \quad\left|z_{0}\right| \geqslant 1
$$

that is, there exists a nonnull vector $V$ such that

$$
\left[\boldsymbol{I}_{\boldsymbol{m}}-\mathscr{K}^{t} \boldsymbol{G}_{k}\left(z_{0}\right)\right] \boldsymbol{V}=0 \text {. }
$$

Equation (47) implies

$$
\boldsymbol{V}^{\dagger} \boldsymbol{V}=\boldsymbol{V}^{\dagger} \boldsymbol{G}_{k}^{\dagger}\left(z_{0}\right) \mathscr{K} \mathscr{K}^{\dagger} \boldsymbol{G}_{k}\left(z_{0}\right) \boldsymbol{V}
$$

Bıt we know, $\mathscr{K} \mathscr{K}^{t}<I_{p}$ (equation (30)) $\left(\boldsymbol{G}_{k}(z)\right.$ being assumed nondegenerate), hence (48) implies

$$
\boldsymbol{V}^{\dagger} \boldsymbol{V}<\boldsymbol{V}^{\dagger} \boldsymbol{G}_{k}^{\dagger}\left(z_{0}\right) \boldsymbol{G}_{k}\left(z_{0}\right) \boldsymbol{V}
$$

Thus by LBR property of $\boldsymbol{G}_{k}(z)$ (Equation (3b)), this 
implies

$$
\boldsymbol{V}^{\dagger} \boldsymbol{V}<\boldsymbol{V}^{\dagger} \boldsymbol{V}
$$

which is a contradiction. This, then, establishes that $\left|z_{0}\right|<1$, i.e., that $G_{k-1}(z)$ is stable and hence LBR.

\section{Completion of Synthesis}

Given a $p \times m$ LBR transfer matrix $G_{N}(z)$, one can repeat the LBR extraction process until the remainder is a constant $p \times m$ matrix $\mathscr{R}$ such that $\mathscr{R}^{t} \mathscr{R}=I_{m}$. This finally gives rise to the terminated cascaded realization of Fig. 4, provided that each of the intermediate LBR transfer matrices $\boldsymbol{G}_{k}(z)$ is nondegenerate.

If, however, $\boldsymbol{G}_{k}(z)$ turns out to be degenerate for some $k$, then the matrices $\left(\boldsymbol{I}_{m}-\mathscr{K}^{t} \mathscr{K}\right)$ and $\left(\boldsymbol{I}_{p}-\mathscr{K} \mathscr{K}^{t}\right)$ are singular. Because of this rank defect, the order reduction procedure described earlier breaks down. Under such a situation, we can obtain a lower dimensional LBR matrix $\boldsymbol{G}_{k}^{(r)}(z)$ of dimension $(p-r) \times(m-r)$ such that $\boldsymbol{G}_{k}^{(r)}(z)$ is nondegenerate. (The procedure for this is described in Appendix A4.) We can then proceed to do the order reduction on $\boldsymbol{G}_{k}^{(r)}(z)$ to obtain the remainder $\boldsymbol{G}_{k-1}(z)$. Thus the cascaded lattice structure now has a different appearance. In Fig. 9 we show an example of this type. Here the $3 \times 3 \mathrm{LBR}$ matrices $G_{4}(z)$ and $G_{3}(z)$ are nondegenerate, but $\boldsymbol{G}_{2}(z)$ is degenerate. The $2 \times 2$ LBR function $G_{2}^{(1)}(z)$, which is extracted from $G_{2}(z)$ as described in Appendix A4, is then synthesized by conventional order reduction techniques. When drawing Fig. 9, it is assumed that $G_{2}^{(1)}(z)$ and $G_{1}(z)$ are nondegenerate.

It should be noticed here that, if an all-pass transfer matrix is $2 \times 1$ (i.e., single-input and two-outputs), then it is nondcgencrate (unless it is a constant). Consequently, the matrices $\left(\boldsymbol{I}_{m}-\mathscr{K}^{t} \mathscr{K}\right)$ and $\left(\boldsymbol{I}_{p}-\mathscr{K}^{t}\right)$ are of full rank throughout the synthesis process. Thus, the $2 \times 1$ all-pass structures reported in [8], [13] are entirely free from degeneracy problems.

\section{A Numerical Example of Lattice Synthesis}

Consider the following 2-input 3-output transfer matrix $(p=3, m=2) G_{3}(z)=N_{3}(z) D_{3}^{-1}(z)$ where

$$
\begin{aligned}
N_{3}(z)= & {\left[\begin{array}{lr}
1.837 & -9.186 \\
0.0 & 12.728 \\
0.919 & -19.290
\end{array}\right]+z^{-1}\left[\begin{array}{rr}
-0.553 & -4.286 \\
4.453 & 14.254 \\
-6.016 & -13.794
\end{array}\right] } \\
& +z^{-2}\left[\begin{array}{rr}
-0.697 & 11.790 \\
6.683 & -5.331 \\
-6.683 & 11.894
\end{array}\right] \\
& +z^{-3}\left[\begin{array}{rr}
2.840 & 5.992 \\
1.500 & -5.786 \\
0.0 & 6.495
\end{array}\right] \\
D_{3}(z)= & {\left[\begin{array}{lr}
2.756 & -13.778 \\
0.0 & 22.045
\end{array}\right]+z^{-1}\left[\begin{array}{rr}
-2.199 & -5.060 \\
7.712 & 18.690
\end{array}\right] } \\
& +z^{-2}\left[\begin{array}{rr}
-1.752 & 13.476 \\
9.020 & -11.028
\end{array}\right] \\
& +z^{-3}\left[\begin{array}{rr}
1.893 & 6.160 \\
0.866 & -7.671
\end{array}\right] .
\end{aligned}
$$

It can be verified that $\boldsymbol{G}_{3}(z)$ is LBR. Note that $\boldsymbol{B}_{N 0}$ is nonsingular, and therefore we can carry out the synthesis procedure successfully ( $\boldsymbol{B}_{N 0}$ is not $\boldsymbol{I}_{2}$ but can be normalized to $I_{2}$ ). The $\mathscr{K}$ parameter of the first stage is, by (27)

$$
\begin{aligned}
\mathscr{K}_{3} & =G_{3}(\infty)=N_{3}(\infty) D_{3}^{-1}(\infty) \\
& =\left[\begin{array}{lc}
0.667 & 0.0 \\
0.0 & 0.577 \\
0.333 & -0.667
\end{array}\right] .
\end{aligned}
$$

After extraction of the appropriate lattice section, the remainder LBR matrix is $G_{2}(z)=N_{2}(z) D_{2}^{-1}(z)$ and can be found from (37a), (37b) to be

$$
\begin{aligned}
\boldsymbol{N}_{2}(z)= & {\left[\begin{array}{ll}
0.444 & 0.222 \\
0.0 & 0.192 \\
0.222 & -0.111
\end{array}\right]+z^{-1}\left[\begin{array}{rr}
0.229 & 0.314 \\
0.656 & 0.467 \\
-0.745 & -0.394
\end{array}\right] } \\
& +z^{-2}\left[\begin{array}{ll}
0.768 & 0.595 \\
0.444 & 0.202 \\
0.0 & 0.108
\end{array}\right] \\
\boldsymbol{D}_{2}(z)= & {\left[\begin{array}{ll}
0.667 & 0.333 \\
0.0 & 0.333
\end{array}\right]+z^{-1}\left[\begin{array}{ll}
0.095 & 0.223 \\
1.136 & 0.719
\end{array}\right] } \\
& +z^{-2}\left[\begin{array}{ll}
0.512 & 0.432 \\
0.257 & 0.045
\end{array}\right] .
\end{aligned}
$$

In a similar manner, we can continue the extraction process. Thus for the next extraction, the $\mathscr{K}$ parameter is

$$
\mathscr{K}_{2}=N_{2}(\infty) D_{2}^{-1}(\infty)=\left[\begin{array}{ll}
0.667 & 0.0 \\
0.0 & 0.577 \\
0.333 & -0.667
\end{array}\right]
$$

and the next LBR-remainder is $G_{1}(z)=N_{1}(z) D_{1}^{-1}(z)$ where

$$
\begin{aligned}
& N_{1}(z)=\left[\begin{array}{ll}
0.333 & 0.333 \\
0.0 & 0.192 \\
0.192 & 0.192
\end{array}\right]+z^{-1}\left[\begin{array}{ll}
0.859 & 0.375 \\
0.544 & 0.103 \\
0.0 & 0.118
\end{array}\right] \\
& D_{1}(z)=\left[\begin{array}{ll}
0.667 & 0.333 \\
0.0 & 0.333
\end{array}\right]+z^{-1}\left[\begin{array}{ll}
0.429 & 0.221 \\
0.744 & 0.281
\end{array}\right]
\end{aligned}
$$

One more extraction is required to complete the synthesis. The $\mathscr{K}$-parameter for the final extraction is

$$
\mathscr{K}_{1}=N_{1}(\infty) D_{1}^{-1}(\infty)=\left[\begin{array}{ll}
0.500 & 0.500 \\
0.0 & 0.577 \\
0.289 & 0.289
\end{array}\right]
$$

After the extraction, we are left with the constant remainder $\mathscr{R}$ that forms the "termination" of the cascade.

$$
\mathscr{R}=\left[\begin{array}{lr}
0.707 & 0.577 \\
0.707 & -0.577 \\
0.0 & 0.577
\end{array}\right]
$$

It can be verified that $\mathscr{R}$ is $\mathrm{LBR}$, i.e., $\mathscr{R}^{t} \mathscr{R}=I_{2}$. The synthesis process is now complete.

\section{Properties of the Recursion Procedure}

In this section, a number of important properties of the recursion procedure are developed. Unless mentioned otherwise, all $\boldsymbol{G}_{k}(z)$ are assumed to be nondegenerate. 


\section{Property 1:}

If the MFD $\boldsymbol{G}_{k}(z)=\boldsymbol{N}_{k}(z) \boldsymbol{D}_{k}^{-1}(z)$ is irreducible, then the $\operatorname{MFD} \boldsymbol{G}_{k-1}(z)=\boldsymbol{N}_{k-1}(z) \boldsymbol{D}_{k-1}^{-1}(z)$ is also irreducible.

Proof: According to "Bezout Identity" [10], a right MFD $\boldsymbol{H}(z)=\boldsymbol{N}(z) \boldsymbol{D}^{-1}(z)$ is irreducible if and only if there exist polynomial matrices $X(z)$ and $Y(z)$ such that

$$
\boldsymbol{X}(z) \boldsymbol{N}(z)+\boldsymbol{Y}(z) \boldsymbol{D}(z)=\boldsymbol{I}_{m} .
$$

Note that all polynomials are "polynomials in $z^{-1}$." Since $\boldsymbol{N}_{k}(z) \boldsymbol{D}_{k}^{-1}(z)$ is irreducible, we, therefore, have

$$
\boldsymbol{X}(z) \boldsymbol{N}_{k}(z)+\boldsymbol{Y}(z) \boldsymbol{D}_{k}(z)=\boldsymbol{I}_{m}
$$

for some $\boldsymbol{X}(z), \boldsymbol{Y}(z)$. But $\boldsymbol{G}_{k}(z)$ and $\boldsymbol{G}_{k-1}(z)$ are related by

$$
\begin{aligned}
& \boldsymbol{N}_{k}(z)=C D_{k-1}(z)+D_{z^{-1}} N_{k-1}(z) \\
& D_{k}(z)=A D_{k-1}(z)+B z^{-1} N_{k-1}(z) .
\end{aligned}
$$

Consequently, the following identity holds:

$$
\begin{aligned}
{[\boldsymbol{X}(z) \boldsymbol{C}} & +\boldsymbol{Y}(z) \boldsymbol{A}] \boldsymbol{D}_{k-1}(z) \\
& +\left[\boldsymbol{X}(z) D z^{-1}+\boldsymbol{Y}(z) B z^{-1}\right] N_{k-1}(z)=I_{m}
\end{aligned}
$$

which shows that $\boldsymbol{N}_{k-1}(z) D_{k-1}^{-1}(z)$ is indeed irreducible.

Property 2:

Let $\boldsymbol{G}_{k}(z)$ be a $p \times m$ LBR transfer matrix, with a right MFD as in (11). Let the $m \times m$ matrix $\boldsymbol{R}(z)$ be a rightcommon divisor of $N_{k}(z)$ and $D_{k}(z)$, i.e., let

$$
\boldsymbol{N}_{k}(z)=\overline{\boldsymbol{N}}_{k}(z) \boldsymbol{R}(z) \quad \boldsymbol{D}_{k}(z)=\overline{\boldsymbol{D}}_{k}(z) \boldsymbol{R}(z)
$$

where $\overline{\boldsymbol{N}}_{k}(z)$ and $\overline{\boldsymbol{D}}_{k}(z)$ are polynomial matrices. Then from (37a) and (37b) it is clear that $\boldsymbol{K}(z)$ is also a right-common divisor of $N_{k-1}(z)$ and $D_{k-1}(z)$. Moreover, according to Property 1 above, if $\bar{N}_{k}(z)$ and $\bar{D}_{k}(z)$ are right-coprime, then the polynomials

$$
\left[\overline{\boldsymbol{N}}_{k}(z)-\mathscr{K} \overline{\boldsymbol{D}}_{k}(z)\right] \text { and }\left[\bar{D}_{k}(z)-\mathscr{K}^{t} \overline{\boldsymbol{N}}_{k}(z)\right]
$$

are right-coprime. Thus if $\boldsymbol{R}(z)$ is a GCRD of $\left(\boldsymbol{N}_{k}, \boldsymbol{D}_{k}\right)$, then it is also a GCRD of the polynomials $\left(N_{k-1}, D_{k-1}\right)$.

Conversely, if $R(z)$ is a GCRD of $N_{k-1}(z)$ and $D_{k-1}(z)$, then it is a GCRD of $N_{k}(z)$ and $D_{k}(z)$ as well. In order to see this, note that (37a), (37b) imply the following:

$$
\begin{aligned}
& \boldsymbol{N}_{k}(z)-\mathscr{K} \boldsymbol{D}_{k}(z)=\left(\boldsymbol{I}_{p}-\mathscr{K} \mathscr{K}^{t}\right)^{1 / 2} z^{-1} \boldsymbol{N}_{k-1}(z) \\
& \boldsymbol{D}_{k}(z)-\mathscr{K}^{t} \boldsymbol{N}_{k}(z)=\left(\boldsymbol{I}_{m}-\mathscr{K}^{t} \mathscr{K}\right)^{1 / 2} \boldsymbol{D}_{k-1}(z) .
\end{aligned}
$$

If $\boldsymbol{R}(z)$ is a right-common divisor of $\boldsymbol{N}_{k-1}(z)$ and $\boldsymbol{D}_{k-1}(z)$, then $\boldsymbol{N}_{k-1}(z) \boldsymbol{R}^{-1}(z)$ and $\boldsymbol{D}_{k-1}(z) \boldsymbol{R}^{-1}(z)$ are matrix polynomials. Hence the following matrices are polynomials:

$$
\mathscr{K}^{t} \boldsymbol{N}_{k}(z) \boldsymbol{R}^{-1}(z)-\mathscr{K}^{t} \mathscr{K} \boldsymbol{D}_{k}(z) \boldsymbol{R}^{-1}(z)
$$

and

$$
\boldsymbol{D}_{k}(z) \boldsymbol{R}^{-1}(z)-\mathscr{K}^{t} \boldsymbol{N}_{k}(z) \boldsymbol{R}^{-1}(z)
$$

Adding these, it is clear that $\left(\boldsymbol{I}_{m}-\mathscr{K}^{t} \mathscr{K}\right) \boldsymbol{D}_{k}(z) \boldsymbol{R}^{-1}(z)$ is a polynomial, hence $\boldsymbol{R}(z)$ is a right divisor of $\boldsymbol{D}_{k}(z)$. Similarly, it can be verified that $\boldsymbol{R}(z)$ is a right divisor of $N_{k}(z)$. By invoking Bezout's Identity again, it can further be verified that if $R(z)$ is a GCRD of $N_{k-1}(z)$ and
$D_{k-1}(z)$, then $D_{k}(z) R^{-1}(z)$ and $N_{k}(z) R^{-1}(z)$ are rightcoprime, hence $\boldsymbol{R}(z)$ is also a GCRD of $D_{k}(z)$ and $N_{k}(z)$.

These results can be summarized as follows:

Property 2: $\boldsymbol{R}(z)$ is a greatest right-common divisor (GCRD) of $N_{k}(z)$ and $D_{k}(z)$ if and only if it is a GCRD of $N_{k-1}(z)$ and $D_{k-1}(z)$.

\section{Property 3: Propagation of the GCRD}

As a consequence of Property 2, given an LBR matrix $\boldsymbol{G}_{N}(z)$, any GCRD $\boldsymbol{R}(z)$ of $\boldsymbol{N}_{N}(z)$ and $\boldsymbol{D}_{N}(z)$ propagates down the line, as the synthesis progresses. Eventually, a stage is reached when we have the following situation:

$$
\boldsymbol{N}_{i}(z)=\mathscr{R} \cdot \boldsymbol{R}(z) \boldsymbol{W}(z), \quad \boldsymbol{D}_{i}(z)=\boldsymbol{R}(z) \boldsymbol{W}(z)
$$

where $\boldsymbol{W}(z)$ is a $m \times m$ unimodular matrix, and $\mathscr{R}$ is a $p \times m$ (constant) matrix, such that $\mathscr{R}^{t} \mathscr{R}=\boldsymbol{I}_{m}$. If we try to perform further LBR extraction, then the lattice parameter is

$$
\mathscr{K}_{i}=\mathscr{R}
$$

hence $\left(\boldsymbol{I}_{m} \mathscr{K}_{i}{ }^{t} \mathscr{K}_{i}\right)$ is null. We do not, therefore, need to proceed any farther, and the synthesis terminates in a "prematured manner." The matrix $\mathscr{R}$ now forms the "termination" of the cascaded lattice.

Thus, given an arbitrary $p \times m$ LBR transfer matrix the above synthesis procedure automatically reveals a GCRD as a by-product. The GCRD can then be used to reduce the given MFD to "minimal form," for other possible applications.

Let us make one further observation. Recall that $D_{N}(z)$ is given by

$$
\boldsymbol{D}_{N}(z)=\left(\boldsymbol{B}_{N 0} z^{N}+\boldsymbol{B}_{N 1} z^{N-1}+\cdots+\boldsymbol{B}_{N N}\right) z^{-N}
$$

Recall also our assumption that $\boldsymbol{B}_{N 0}$ is nonsingular. In particular, none of the columns of $\boldsymbol{B}_{N 0}$ can be null, hence, the column-degree of each column in $\boldsymbol{D}_{N}(z)$ is equal to $N$. The nonsingularity of $\boldsymbol{B}_{N 0}$ also implies that $\boldsymbol{D}_{N}(z)$ is column reduced [10], hence,

$$
\operatorname{deg} \operatorname{det} \boldsymbol{D}_{N}(z)=\sum_{i=1}^{m} N=m N \text {. }
$$

With $R(z)$ the GCRD of $N_{N}(z)$ and $D_{N}(z)$ of a given LBR transfer matrix, we thus have

$$
\begin{aligned}
\operatorname{deg} \operatorname{det} \boldsymbol{D}_{N}(z) & =m N \\
& =\operatorname{deg} \operatorname{det} \overline{\boldsymbol{D}}_{N}(z)+\operatorname{deg} \operatorname{det} \boldsymbol{R}(z) .
\end{aligned}
$$

Let $R(z)$ be of the form

$$
\boldsymbol{R}(z)=\boldsymbol{R}_{0}+\boldsymbol{R}_{1} z^{-1}+\cdots+\boldsymbol{R}_{N_{1}} z^{-N_{1}} .
$$

By the assumption that $\boldsymbol{B}_{N O}$ is nonsingular and that all intermediate LBR functions $\boldsymbol{G}_{k}(z)$ are nondegenerate, it is clear that $\boldsymbol{R}_{0}$ is nonsingular (see (38) and discussions thereof). Thus $\boldsymbol{R}(z)$ is column-reduced and hence deg det $\boldsymbol{R}(z)=m N_{1}$. Moreover, in view of the above form of $\boldsymbol{R}(z)$, it is clear that (at most) $N-N_{1}$ steps of orderreduction have taken place thus far. Hence the number of delay units in a cascade of the form of Fig. 1 is at most equal to $\left(N-N_{1}\right) m$. From (65) it is also clear that 


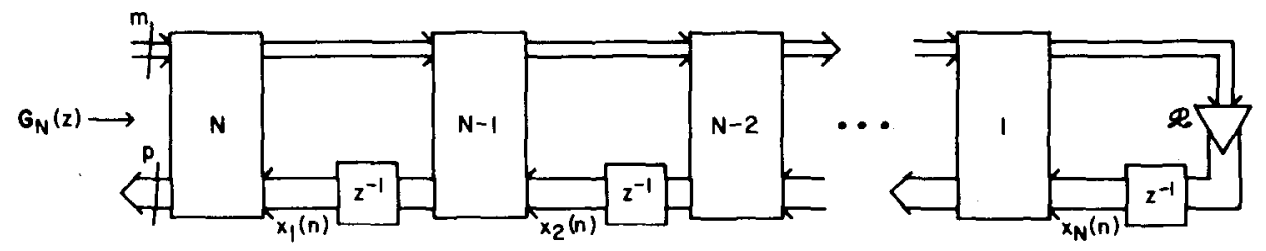

Fig. 7. Pertaining to the state observability property.

the McMillan degree of $G_{N}(z)$ is

$$
\operatorname{deg} \operatorname{det} \overline{\boldsymbol{D}}_{N}(z)=\left(N-N_{1}\right) m \text {. }
$$

In summary, the number of delays in Fig. 1 is precisely equal to the McMillan degree of $\boldsymbol{G}_{N}(z)$. In other words, even if the given LBR transfer matrix $G_{N}(z)$ is not irreducible, the synthesis procedure automatically gives rise to a minimal realization because of prematured tcrmination.

The next property pertains to an equivalent realization of Fig. 4. By moving the delay units in Fig. 4 from the top lines to the bottom lines, the obviously equivalent realization of Fig. 7 is obtained. Unlike Fig. 4, Fig. 7 however represents a nonminimal realization (unless $p=m$ ). However, it still turns out to be a completely observable realization.

\section{Property 4:}

The lattice realization of Fig. 7 for the $p \times m$ LBR transfer matrix $\boldsymbol{G}_{N}(z)$ is completely observable, even if $\boldsymbol{G}_{N}(z)=\boldsymbol{N}_{N}(z) \boldsymbol{D}_{N}^{-1}(z)$ is not in irreducible form. (Moreover, if $p=m$, then Fig. 7 represents a minimal realization.)

Proof: With state-variables as indicated in Fig. 7, the overall system can be described by

$$
\begin{gathered}
\boldsymbol{x}(n+1)=\mathscr{A} \boldsymbol{x}(n)+\mathscr{B} \boldsymbol{u}(n) \\
\boldsymbol{y}(n)=\mathscr{C} \boldsymbol{x}(n)+\mathscr{D} \boldsymbol{u}(n)
\end{gathered}
$$

where $\boldsymbol{x}(n)$ is a $N p$-vector defined as

$$
\boldsymbol{x}(n)=\left[\begin{array}{lll}
\boldsymbol{x}_{\mathbf{1}}^{t}(n) & \cdots & \boldsymbol{x}_{N}^{t}(n)
\end{array}\right]^{t}
$$

with $x_{k}(n)$ being $p$-vectors, as indicated in the figure. The state matrices are given by

$$
\mathscr{A}=\left[\begin{array}{cccccc}
\boldsymbol{A}_{11} & \boldsymbol{A}_{12} & 0 & 0 & \cdots & 0 \\
\boldsymbol{A}_{21} & \boldsymbol{A}_{22} & \boldsymbol{A}_{23} & 0 & \cdots & 0 \\
\vdots & & \ddots & & & \vdots \\
\vdots & & & \ddots & & \vdots \\
\vdots & & & & \ddots & \vdots \\
\boldsymbol{A}_{N-1,1} & \cdots & \cdots & \cdots & \cdots & \boldsymbol{A}_{N-1, N} \\
\boldsymbol{A}_{N 1} & \cdots & \cdots & \cdots & \cdots & \boldsymbol{A}_{N, N}
\end{array}\right]
$$

and

$$
\mathscr{C}=\left[\begin{array}{llll}
C_{1} & 0 & \cdots & 0
\end{array}\right]
$$

The exact forms of the matrices $\mathscr{B}$ and $\mathscr{D}$ are not of interest here. Each $\boldsymbol{A}_{i j}$ is $p \times p$ and so is $\boldsymbol{C}_{1}$. The crucial point is that the following matrices:

$$
A_{12}, A_{23}, A_{34}, \cdots, A_{N-1, N}
$$

which occur on the upper block-diagonal of $\mathscr{A}$ are of the form:

$$
\boldsymbol{A}_{i-1, i}=\left(\boldsymbol{I}_{p}-\mathscr{K}_{j} \mathscr{K}_{j}^{t}\right)^{1 / 2}, \quad j=N+1-i
$$

where $\mathscr{K}_{j}$ is the $\mathscr{K}$-parameter of the $j$ th LBR matrix two-pair. These matrices are, therefore, nonsingular. Moreover,

$$
C_{1}=\left(I_{p}-\mathscr{K}_{N} \mathscr{K}_{N}^{t}\right)^{1 / 2}
$$

is nonsingular too. The "PBH-rank test," which is stated below for convenience, then reveals that $\{\mathscr{C}, \mathscr{A}\}$ is completely observable.

The Popov-Belevitch-Hautus (PBH) Test [10]: The pair $\{\mathscr{C}, \mathscr{A}\}$ is completely observable if and only if there does not exist an eigenvector of $\mathscr{A}$ orthogonal to all rows of $\mathscr{C}$.

Indeed, let $V=\left[\begin{array}{lll}V_{1}^{t} V_{2}^{t} & \cdots & V_{N}^{t}\end{array}\right]^{t}$ be a vector such that

$$
\mathscr{A} \boldsymbol{V}=\lambda \boldsymbol{V}
$$

and

$$
\mathscr{C} \boldsymbol{V}=\mathbf{0} .
$$

Because of the nonsingularity of $\boldsymbol{C}_{1}$, (77) implies that $V_{1}=\mathbf{0}$. Consequently, (76) implies

$$
A_{12} V_{2}=0
$$

and by nonsingularity of $\boldsymbol{A}_{12}$, we have $\boldsymbol{V}_{2}=\mathbf{0}$. Continuing in this manner, it is clear that $\boldsymbol{V}=\mathbf{0}$. Thus there does not exist a nonnull vector satisfying (76) and (77), which proves that $\{\mathscr{C}, \mathscr{A}\}$ is completely observable.

\section{Synthesis of Arbitrary Transfer Matrices}

Given an arbitrary $k \times m$ transfer matrix that is not LBR, $\quad H(z)=N(z) D^{-1}(z)$ assume that it satisfies $\boldsymbol{H}^{\dagger}\left(e^{j \omega}\right) \boldsymbol{H}\left(e^{j \omega}\right) \leqslant \boldsymbol{I}_{m}$ for all $\boldsymbol{\omega}$. (This can be achieved by scaling with a scalar constant.) Since $\boldsymbol{H}(z)$ is not LBR, we cannot directly employ the cascaded-lattice synthesis procedure described earlier. However, we can construct an LBR transfer matrix, $\boldsymbol{G}_{N}(z)$ such that synthesis of $\boldsymbol{G}_{N}(z)$ leads also to the synthesis of $\boldsymbol{H}(z)$. Thus define a new transfer matrix

$$
\boldsymbol{G}_{N}(z)=\left[\begin{array}{l}
\boldsymbol{N}(z) \\
\boldsymbol{n}(z)
\end{array}\right] \boldsymbol{D}^{-1}(z)
$$

where $\boldsymbol{n}(z)$ is a matrix polynomial such that

$$
\tilde{\boldsymbol{n}}(z) \boldsymbol{n}(z)=\tilde{\boldsymbol{D}}(z) \boldsymbol{D}(z)-\tilde{\boldsymbol{N}}(z) \boldsymbol{N}(z) .
$$

Clearly, $\boldsymbol{G}_{N}(z)$ is LBR. The existence of $\boldsymbol{n}(z)$ follows from the well-known results due to Youla [16], on matrix spectral factorization. The matrix $\boldsymbol{n}(z)$ is $r \times m$ where $r$ is the "normal rank" of the polynomial on the right-hand side of (80). We have essentially "embedded" $\boldsymbol{H}(z)$ into $\boldsymbol{G}_{N}(z)$, 
which is $p \times m$ LBR $(p=r+k)$, and can be synthesized in the form of a terminated cascaded-lattice. Then the first $k$ outputs of $\boldsymbol{G}_{N}(z)$ are taken as the system output, and the remaining $r$ outputs are ignored.

It is worth noting that the lattice-synthesis procedure can be used for testing the stability of an arbitrary $k \times m$ transfer matrix $\boldsymbol{H}(z)$. For this, we form an allpass matrix $\boldsymbol{G}_{N}(z)$ from $\boldsymbol{H}(z)$ as described above and then synthesize a lattice. The matrix $\boldsymbol{H}(z)$ is stable if $\boldsymbol{G}_{N}(z)$ is stable (i.e., LBR) which in turn is true if the $\mathscr{K}$ parameters of the lattice-structure satisfy

$$
\mathscr{K}_{i} \mathscr{K}_{i}<I_{m}
$$

for all $i$. In fact, (81) is not necessary for stability because a possible equality in (81) might occur with degenerate allpass matrices. Details of the stability test procedure are however more involved, and are outside the scope of this paper.

\section{Summary and Concluding Remarks}

Starting from the right-MFD description of a $p \times m$ LBR transfer matrix (equation (15)), we have obtained two equivalent cascaded-lattice structures, as shown in Figs. 4 and 7. These structures are meaningful as long as none of the intermediate allpass matrices $\boldsymbol{G}_{k}(z)$ is degenerate. (The case when some of the $\boldsymbol{G}_{k}(z)$ are degenerate allpass functions is handled by decoupling the memoryless direction and reducing the problem dimension as shown in Appendix A4.) An assumption we made is that the polynomial matrix $D_{N}(z)$ (Equation (16)) is "regular," i.e., the leading coefficient $\boldsymbol{B}_{N 0}$ nonsingular. The "lattice parameter matrices" $\mathscr{K}_{i}$ satisfy the conditions of (29) and (30), and consequently, each lattice two-pair is a $(p+m) \times(p+m)$ real orthogonal matrix.

The realization of Fig. 1 is minimal, whereas that of Fig. 7 is "completely observable." Moreover, the denominator $\boldsymbol{D}_{N}(z)$ of the irreducible MFD is automatically "columnreduced," and, therefore, the determinantal degree of $\boldsymbol{D}_{N}(z)$ is precisely $m N$, which is the order of the controllable realization of Fig. 4. Thus Fig. 4 is a minimal realization for an irreducible LBR MFD. In view of the fact that $p \geqslant m$ for an LBR transfer matrix, the realization of Fig. 7 is minimal if and only if $p=m$ and the given MFD is irreducible. The realization of Fig. 4 reduces to the normalized lattice structure for $p=m=1$ and reduces to certain known orthogonal digital filter structures for $p=2, m=1$.

When LBR transfer matrices with nonminimal MFDs are synthesized using the procedures outlined, the GCRD in the MFD is automatically revealed, and the controllable realization of Fig. 1 is in fact still a minimal realization. Finally, given an arbitrary (non-LBR) stable transfer matrix, we have outlined a procedure by which a cascadedlattice realization can be obtained. In addition, a procedure for testing the stability of a transfer matrix emerges as a byproduct of the synthesis theory.

\section{APPENDIX A1}

Let $\boldsymbol{H}(z)$ be a $p \times m$ LBR transfer matrix. Then, at any frequency, say $\omega=0$, we have

$$
\boldsymbol{H}^{\prime}\left(e^{j 0}\right) \boldsymbol{H}\left(e^{j 0}\right)=\boldsymbol{I}_{\boldsymbol{m}} .
$$

The matrix $\boldsymbol{H}\left(e^{j 0}\right)$ cannot have a row or column rank exceeding $p$, hence if $p<m$, (A1) cannot be satisfied. This shows $p \geqslant m$ for any $p \times m$ LBR matrix.

\section{APPENDIX A2}

Proof of INEQuality ( $3 b$ )

Let $\boldsymbol{H}(z)$ be any $p \times m$ LBR transfer matrix. We then have by definition:

$$
\boldsymbol{H}^{\dagger}\left(e^{j \omega}\right) \boldsymbol{H}\left(e^{j \omega}\right)=\boldsymbol{I}_{m}, \quad \text { for all } \boldsymbol{\omega} .
$$

If $\boldsymbol{x}(n)$ is an input sequence ( $m$-vector) and $y(n)$ the corresponding output sequence ( $p$-vector) then (A2) implies

$$
\boldsymbol{Y}^{\dagger}\left(e^{j \omega}\right) \boldsymbol{Y}\left(e^{j \omega}\right)=\boldsymbol{X}^{\dagger}\left(e^{j \omega}\right) \boldsymbol{X}\left(e^{j \omega}\right)
$$

Integrating and applying Parseval's relation leads to the equality:

$$
\sum_{n=-\infty}^{\infty} \boldsymbol{y}^{\dagger}(n) \boldsymbol{y}(n)=\sum_{n=-\infty}^{\infty} \boldsymbol{x}^{\dagger}(n) \boldsymbol{x}(n)
$$

for any finite-energy input.

Let us apply the following input:

$$
\boldsymbol{x}(n)= \begin{cases}z_{0}^{n} \boldsymbol{V}, & -\infty \leqslant n \leqslant M \\ 0, & n>M\end{cases}
$$

where $M$ is any integer, $V$ is any $m$-vector, and $\left|z_{0}\right|>1$. Since $\boldsymbol{H}(z)$ is defined for all $|z|>1$, we thus have

$$
\boldsymbol{y}(n)= \begin{cases}z_{0}^{n} \boldsymbol{H}\left(z_{0}\right) \boldsymbol{V}, & -\infty \leqslant n \leqslant M \\ \text { something else, } & n>M .\end{cases}
$$

We, therefore, have

$$
\begin{aligned}
\sum_{n=-\infty}^{M} \boldsymbol{y}^{\dagger}(n) \boldsymbol{y}(n) & =\boldsymbol{V}^{\dagger} \boldsymbol{H}^{\dagger}\left(z_{0}\right) \boldsymbol{H}\left(z_{0}\right) \boldsymbol{V} \sum_{n=-\infty}^{M}\left|z_{0}\right|^{2 n} \\
\leqslant \sum_{n=-\infty}^{\infty} \boldsymbol{y}^{\dagger}(n) \boldsymbol{y}(n) & =\sum_{n=-\infty}^{\infty} \boldsymbol{x}^{\dagger}(n) \boldsymbol{x}(n) \\
& =\boldsymbol{V}^{\dagger} \boldsymbol{V} \sum_{n=-\infty}^{M}\left|z_{0}\right|^{2 n}
\end{aligned}
$$

Thus the following inequality:

$$
\boldsymbol{V}^{\dagger} \boldsymbol{H}^{\dagger}\left(z_{0}\right) \boldsymbol{H}\left(z_{0}\right) \boldsymbol{V} \leqslant \boldsymbol{V}^{\dagger} \boldsymbol{V}
$$

holds for all $V$, which shows

$$
\boldsymbol{H}^{\dagger}\left(z_{0}\right) \boldsymbol{H}\left(z_{0}\right) \leqslant \boldsymbol{I}_{m}, \quad \text { for }\left|z_{0}\right|>1 .
$$

Note that if $\boldsymbol{H}(z)$ were BR rather than LBR, i.e., if $\boldsymbol{H}^{\dagger}\left(e^{j \omega}\right) \boldsymbol{H}\left(e^{j \omega}\right) \leqslant \boldsymbol{I}_{m}$, then the inequality of (A9) still holds.

Let us now consider the possibility of equality in (A8). If (A8) is an equality for some $\boldsymbol{V} \neq \mathbf{0}$, some $z_{0}\left(\left|z_{0}\right|>1\right)$, and some $M$ in (A5), then this implies that $y(n)$ is identically 
zero for $n>M$ in (A6). (For, otherwise, the inequality in (A7) would be strict.) Now, $y(n)$ can be written as

$$
\boldsymbol{y}(n)=\sum_{m=-\infty}^{n} \boldsymbol{h}(n-m) \boldsymbol{x}(m)
$$

where $\boldsymbol{h}(\boldsymbol{n})$ represents the (causal) impulse response matrix corresponding to $\boldsymbol{H}(z)$. Since $\boldsymbol{x}(m)=0$ for $m>M$, it is clear that equality in (A8) implies

$$
\begin{gathered}
\boldsymbol{y}(n)-\mathbf{0}=\sum_{m=-\infty}^{M} \boldsymbol{h}(n-m) \boldsymbol{x}(m) \\
\boldsymbol{y}(n+1)=\mathbf{0}=\sum_{m=-\infty}^{M} \boldsymbol{h}(n+1-m) \boldsymbol{x}(m)
\end{gathered}
$$

for $n>M$. Subtracting (A11) from (A12), and remembering that $\boldsymbol{x}(n)$ is as in (A5), we arrive at the result

$$
\boldsymbol{h}(n) \boldsymbol{V}=\mathbf{0}, \quad n>0 .
$$

In other words, $\boldsymbol{V}$ is orthogonal to all the impulse response coefficients except possibly $\boldsymbol{h}(0)$. As a result, $\boldsymbol{H}(z) \boldsymbol{V}=$ $\boldsymbol{h}(0) \boldsymbol{V}=$ constant. In summary, if there exists $\boldsymbol{V} \neq \mathbf{0}$ such that (A8) holds with equality for some $z_{0}$ outside the unit circle, then $\boldsymbol{H}(z) \boldsymbol{V}=\boldsymbol{h}(0) \boldsymbol{V}=$ constant for all $z$, identically. In particular, $\boldsymbol{H}(\infty) \boldsymbol{V}=\boldsymbol{h}(0) \boldsymbol{V}$ and $\boldsymbol{V}^{\dagger} \boldsymbol{H}^{\dagger}(z) \boldsymbol{H}(z) \boldsymbol{V}=\boldsymbol{V}^{\dagger} \boldsymbol{V}$ for all $z$, for this particular $V$.

The condition $\boldsymbol{H}(z) \boldsymbol{V}=$ constant can be expressed in words as " $\boldsymbol{H}(z)$ is memoryless in the direction $\boldsymbol{V}$." Basically, this means that if we apply an input vector sequence of the form $\boldsymbol{x}(n)=s(n) \boldsymbol{V}$ where $s(n)$ is scalar, then the output sequence $\boldsymbol{y}(n)$ at time $n$ depends only on $\boldsymbol{x}(n)$ and not on past values $\boldsymbol{x}(n-k), k>0$. A transfer matrix that is not memoryless in any direction is termed nondegenerate. Otherwise we call it degenerate. We summarize these results as follows:

Let the $p \times m$ transfer matrix $\boldsymbol{H}(z)$ be BR. Then $\boldsymbol{H}^{\dagger}(z) \boldsymbol{H}(z) \leqslant \boldsymbol{I}_{m}$ for all $z$ such that $|z| \geqslant 1$. Moreover, if there exists a vector $V \neq 0$ such that

$$
\boldsymbol{V}^{\dagger} \boldsymbol{H}^{\dagger}(z) \boldsymbol{H}(z) \boldsymbol{V}=\boldsymbol{V}^{\dagger} \boldsymbol{V}
$$

for some $z$ with $|z|>1$, then $\boldsymbol{H}(z) \boldsymbol{V}=$ constant for all $z$, and (A14) holds for all $z$, for this $\boldsymbol{V}$. Consequently, if $\boldsymbol{H}(z)$ is BR and nondegenerate, then (A9) holds with strict inequality.

\section{APPENDIX A3}

ProOF of (19) AND (20)

Referring to Fig. 2, by definition of the chain parameters:

$$
\begin{aligned}
& X_{1}(z)=A(z) Y_{2}(z)+B(z) X_{2}(z) \\
& Y_{1}(z)=C(z) Y_{2}(z)+D(z) X_{2}(z)
\end{aligned}
$$

Let $\left\{\boldsymbol{X}_{11}(z), \boldsymbol{X}_{12}(z), \cdots, \boldsymbol{X}_{1 m}(z)\right\}$ be a set of input vectors, applied (one at a time), in place of $\boldsymbol{X}_{1}(z)$. Let $\left\{\boldsymbol{X}_{21}(z), \cdots, \boldsymbol{X}_{2 m}(z)\right\}$ be the corresponding set of inputs in place of $\boldsymbol{X}_{2}(z)$. Let $\left\{\boldsymbol{Y}_{11}(z), \cdots, \boldsymbol{Y}_{1 m}(z)\right\}$ and $\left\{\boldsymbol{Y}_{21}(z), \cdots, \boldsymbol{Y}_{2 m}(z)\right\}$ denote the corresponding outputs in place of $Y_{1}(z)$ and $Y_{2}(z)$, respectively. Define the matrices:

$$
\begin{aligned}
& \mathscr{X}_{1}(z)=\left[\boldsymbol{X}_{11}(z) \boldsymbol{X}_{12}(z), \cdots, \boldsymbol{X}_{1 m}(z)\right] \\
& \mathscr{Y}_{1}(z)=\left[\boldsymbol{Y}_{11}(z) \boldsymbol{Y}_{12}(z), \cdots, \boldsymbol{Y}_{1 m}(z)\right] \\
& \mathscr{X}_{2}(z)=\left[\boldsymbol{X}_{21}(z) \boldsymbol{X}_{22}(z), \cdots, \boldsymbol{X}_{2 m}(z)\right] \\
& \mathscr{Y}_{2}(z)=\left[\boldsymbol{Y}_{21}(z) \boldsymbol{Y}_{22}(z), \cdots, \boldsymbol{Y}_{2 m}(z)\right] .
\end{aligned}
$$

Then the following equations are true:

$$
\begin{aligned}
& \mathscr{X}_{1}(z)=\boldsymbol{A}(z) \mathscr{Y}_{2}(z)+\boldsymbol{B}(z) \mathscr{X}_{2}(z) \\
& \mathscr{Y}_{1}(z)=\boldsymbol{C}(z) \mathscr{Y}_{2}(z)+\boldsymbol{D}(z) \mathscr{X}_{2}(z) .
\end{aligned}
$$

Now it is clear from Fig. 5 that $\mathscr{X}_{2}(z)$ and $\mathscr{Y}_{2}(z)$ are related as

$$
\mathscr{X}_{2}(z)=\hat{N}_{k}(z) \hat{D}_{k}^{-1}(z) \mathscr{Y}_{2}(z)
$$

Thus $(\Lambda 21)$ and $(\Lambda 22)$ become

$$
\begin{aligned}
& \mathscr{X}_{1}(z)=\left[\boldsymbol{A}(z)+\boldsymbol{B}(z) \hat{\boldsymbol{N}}_{k}(z) \hat{\boldsymbol{D}}_{k}^{-1}(z)\right] \mathscr{Y}_{2}(z) \\
& \mathscr{Y}_{1}(z)=\left[\boldsymbol{C}(z)+\boldsymbol{D}(z) \hat{N}_{k}(z) \hat{D}_{k}^{-1}(z)\right] \mathscr{Y}_{2}(z) .
\end{aligned}
$$

If we now pick $\mathscr{X}_{1}(z)=D_{k}(z)$ then

$$
\mathscr{Y}_{1}(z)=G_{k}(z) \mathscr{X}_{1}(z)=N_{k}(z) D_{k}^{-1}(z) D_{k}(z)=N_{k}(z)
$$

whence (A24) becomes

$$
\begin{aligned}
& \boldsymbol{D}_{k}(z)=\left[\boldsymbol{A}(z) \hat{\boldsymbol{D}}_{k}(z)+\boldsymbol{B}(z) \hat{\boldsymbol{N}}_{k}(z)\right] \hat{\boldsymbol{D}}_{k}^{-1}(z) \mathscr{Y}_{2}(z) \\
& \boldsymbol{N}_{k}(z)=\left[\boldsymbol{C}(z) \hat{\boldsymbol{D}}_{k}(z)+\boldsymbol{D}(z) \hat{\boldsymbol{N}}_{k}(z)\right] \hat{\boldsymbol{D}}_{k}^{-1}(z) \mathscr{Y}_{2}(z)
\end{aligned}
$$

which establishes (19) and (20) within an arbitrary common right-divisor matrix.

\section{APPENDIX A4}

Let $\boldsymbol{G}_{k}(z)$ be a degenerate $p \times m$ LBR transfer matrix that is memoryless in the direction $V$, i.e., $\boldsymbol{G}_{k}(z) \boldsymbol{V}=$ constant $=c$. Assume $\boldsymbol{V}^{\dagger} \boldsymbol{V}=1$ without loss of generality. Now consider the new $p \times m$ matrix

$$
\boldsymbol{H}_{k}(z)=\boldsymbol{G}_{k}(z) \boldsymbol{S}
$$

where $S$ is an $m \times m$ orthogonal matrix, whose first column is $\boldsymbol{V}$. Clcarly, $\boldsymbol{H}_{k}(z)$ continues to be $p \times m$ LBR. Moreover, $\boldsymbol{H}_{k}(z)$ is in the form

$$
\boldsymbol{H}_{k}(z)=\left[\begin{array}{cc}
\boldsymbol{c} & \boldsymbol{F}_{k}(z)
\end{array}\right]
$$

where $c^{\dagger} c=1$. Let us now define a $p \times p$ orthogonal matrix $\boldsymbol{R}$ with first row equal to $\boldsymbol{c}^{\dagger}$

$$
\boldsymbol{R}=\left[\begin{array}{l}
\boldsymbol{c}^{\dagger} \\
\boldsymbol{C}
\end{array}\right]
$$




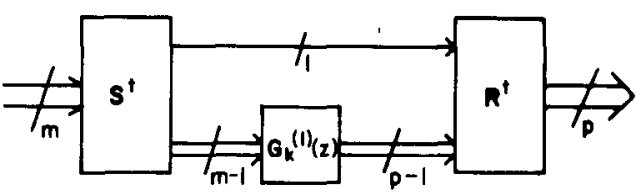

Fig. 8. Pertaining to decoupling of the memoryless direction.

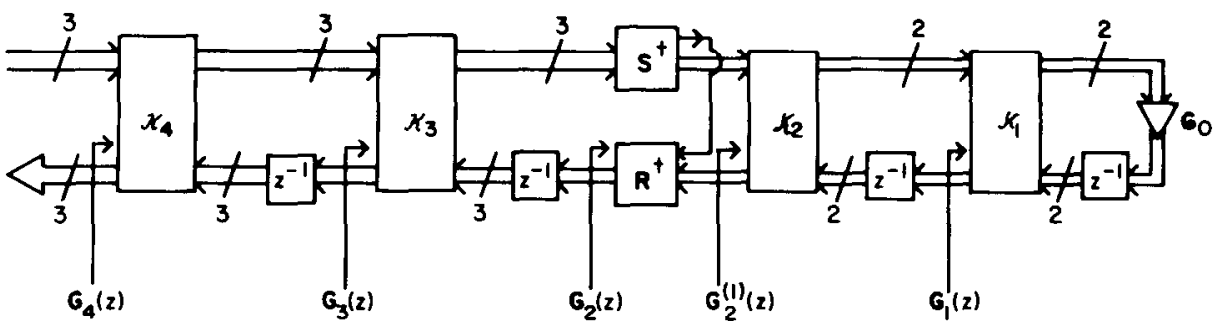

Fig. 9. An example of demonstrating defective rank cases.

Clearly $\boldsymbol{R H}_{k}(z)$ is also $p \times m$ LBR, and we have

$$
\begin{aligned}
\boldsymbol{R H}_{k}(z) & =\boldsymbol{R} \boldsymbol{G}_{k}(z) \boldsymbol{S}=\left[\begin{array}{l}
\boldsymbol{c}^{\dagger} \\
\boldsymbol{C}
\end{array}\right]\left[\boldsymbol{c F}_{k}(z)\right] \\
& =\left[\begin{array}{cc}
1 & \mathbf{0} \\
\mathbf{0} & \boldsymbol{G}_{k}^{(1)}(z)
\end{array}\right] .
\end{aligned}
$$

Thus

$$
\boldsymbol{G}_{k}(z)=\boldsymbol{R}^{t}\left[\begin{array}{cc}
1 & \mathbf{0} \\
\mathbf{0} & \boldsymbol{G}_{k}^{(1)}(z)
\end{array}\right] \boldsymbol{S}^{t}
$$

So, $\boldsymbol{G}_{k}(z)$ can be obtained by synthesizing the $(p-1) \times$ $(m-1)$ LBR transfer matrix $\boldsymbol{G}_{k}^{(1)}(z)$ and making the interconnection shown in Fig. 8. Thus the dimensionality of the synthesis problem has been reduced, by "decoupling". the "memoryless" portion of $\boldsymbol{G}_{k}(z)$. If $\boldsymbol{G}_{k}^{(1)}(z)$ is also degenerate, we can then repeat this process until we eventually arrive at a nondegenerate all-pass matrix $\boldsymbol{G}_{k}^{(r)}(z)$.

\section{ACKNOWLEDGMEN}

We wish to express our appreciation to Reviewer \#2, who not only pointed out some important references, but also suggested a number of very valuable improvements to the manuscript.

\section{REFERENCES}

[1] A. H. Gray, Jr. and J. D. Markel, "A normalized digital filter structure," IEEE Trans. Acoust. Speech, Signal Processing, vol. ASSP-23, pp. 268-277, June 1975 .

[2] J. D. Markel and A. H. Gray, Jr., "Roundoff noise characteristics of a class of orthogonal polynomial structures," IEEE Trans. A coust., Speech, Signal Processing, vol. ASSP-23, pp. 473-485, Oct. 1975.

[31 G. Szego, Orthogonal Polynomial. New York: American Math Soc., Coll., 1959

[4] T. Kailath, "A view of three decades of linear filtering theory," IEEE Trans. Inform. Theory, vol. IT-20, pp. 146-181, Mar. 1974

[5] R. A. Wiggins and E. A. Robinson, "Recursive solution to the multichannel filtering problem," J. Geophys. Res., vol. 70, no. 8, pp. 1885-1891, 1965.

[6] B. D. O. Anderson and J. B. Moore, Optimal Filtering. Englewood Cliffs, NJ: Prentice Hall, 1979

[7] P. Ananthakrishna, B. D. O. Anderson, and S. K., Mitra, "Realization of digital transfer functions using cascaded lattice and ladder block structures," J. Franklin Inst., vol. 316, pp. 93-124, July 1983.

[8] D. Henrot and C. T. Mullis, "A modular and orthogonal digital filter structure for parallel processing," in Proc. IEEE Int. Conf. on Acoustics, Speech, and Signal Processing, Boston, MA, pp. 623-626, Apr. 1983.
[9] P. Delsarte, Y. Genin, and Y, Kamp, "On the mathematical foundation of the generalized Levinson algorithm," in Proc. IEEE Int Conf. on Acoust., Speech, Signal Processing, Paris, France, pp. 1717-1720, 1982.

[10] T. Kailath, Linear Systems, Englewood Cliffs, NJ: Prentice Hall, 1980.

[11] P. P. Vaidyanathan and S. K. Mitra, "Low passband sensitivity digital filters: A generalized viewpoint and synthesis procedures," Proc. IEEE, vol. 72, pp. 404-423, Apr. 1984.

[12] P. Delsarte, Y. V. Genin, and Y. G. Kamp, "Orthogonal polynomial matrices on the unit circle," IEEE Trans. Circuits Syst., vol. CAS-25, pp. 149-160, Mar. 1978.

[13] Sailesh K. Rao and T. Kailath, "Orthogonal digital lattice filters for VLSI implementation," IEEE Trans. Circuits Syst., vol. CAS-31, pp. 933-945, Nov. 1984.

[14] B. D. O. Anderson and S. Vongpanitlerd, Network Analysis and Synthesis. Englewood Cliffs, NJ: Prentice Hall, 1973.

[15] F. R. Gantmacher, The Theory of Matrices. New York: Chelsa 1977.

[16] D. C. Youla, "On the factorization of rational matrices," IRE Trans. Inform. Theory, vol. IT-7, pp. 172-189, July 1961.

[17] C. A. Desoer and J. D. Schulman, "Zeros and poles of matrix transfer functions and their dynamical interpretation," IEEE Trans. Circuits Syst., vol. CAS-21, pp. 3-8, Jan. 1974.

[18] S. K. Mitra, P. S. Kamal, and D. C. Huey, "Cascaded lattice realization of digital filters," Int. J. Circuit Theory Appl., vol. 5, pp. $3-11,1977$.

[19] E. Deprettcre and P. DeWilde, "Orthogonal cascadc realization of real multiport digital filters," Int. J. Circuit Theory Appl., vol. 8, pp. $245-277,1980$

[20] V. P. Potapov, "The multiplicative structure of J-Contractive matrix functions," Amer. Math. Soc., Translation Series 2, vol. 15, pp. $131-243,1960$.

[21] V. Belevitch, Classical Network Theory. San Francisco, CA: Holden Day, 1968.

[22] P. Delsarte, Y. Genin, and Y. Kamp, "On the role of the Nevanlinna-Pick problem in circuit and system theory," Int. J. Circuit Theory Appl., vol. 9, pp. 177-187, 1981.

[23] P. Dewilde and H. Dym, "Schur recursions, error formulas and convergence of rational estimators for stationary stochastic sequences," IEEE Trans. Inform. Theory, vol. IT-27, pp. 446-461, July 1981

[24] P. Dewilde and H. Dym, "Lossless inverse scattering, digital filters and estimation theory," IEEE Trans. Inform. Theory, vol. IT-30, pp. 644-662, July 1984.

\section{幽}

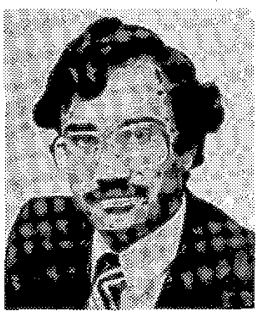

P. P. Vaidyanalhan (S'80)-M'83) was born in Calcutta India, on October 16,1954 . He received the B.Sc. (hons.) degree in physics, and B.Tech. and M.Tech. degrees in radiophysics and electronics from the University of Calcutta, India, in 1974,1977 , and 1979, respectively, and the Ph.D. degree in electrical and computer engineering from the University of California, Santa Barbara, in 1982.

He was a Post-Doctoral Fellow at the University of California, Santa Barbara, from Septem- 
ber 1982 to February 1983. Since March 1983, he has been with the California Institute of Technology, Pasadena, as an Assistant Professor of Electrical Engineering. He was recipient of the award for Excellence in Teaching at the California Institute of Technology, for the year 1983-1984. His main rescarch intcrests arc in digital signal processing, linear systems and filter design.

Dr. Vaidyanathan currently serves as an Associate Editor for the IEEE Transactions on Circuits and SySTEMS. He also served as the Vice-
Chairman of the Technical Program Committee for the 1983 IEEE International Symposium on Circuits and Systems.

幽

Sanjit K. Mitra (S'59-M'63-SM'69-F'74), for a photograph and biography please see page 224 of the March 1985 issue of this TransaCtions. 